\title{
Inhibition of p38 mitogen-activated protein kinase attenuates left ventricular dysfunction by mediating pro-inflammatory cardiac cytokine levels in a mouse model of diabetes mellitus
}

\author{
D. Westermann $\cdot$ S. Rutschow $\cdot$ S. Van Linthout . \\ A. Linderer • C. Bücker-Gärtner • M. Sobirey • A. Riad • \\ M. Pauschinger $\cdot$ H.-P. Schultheiss $\cdot$ C. Tschöpe
}

Received: 16 April 2006 / Accepted: 3 July 2006 / Published online: 26 August 2006

(C) Springer-Verlag 2006

\begin{abstract}
Aims/hypothesis We investigated the effect of SB 203580, a pharmacological inhibitor of p38 mitogen-activated protein kinase (MAPK), on cardiac inflammation, cardiac fibrosis, and left ventricular function using an animal model of diabetic cardiomyopathy.

Materials and methods Diabetes mellitus was induced by streptozotocin (50 mg/kg i.p. for 5 days) in 20 C57/BL6J mice. Diabetic mice were treated daily with the p38 MAPK inhibitor SB 203580 ( $1 \mathrm{mg} / \mathrm{kg}$ daily, $n=10)$ or with placebo $(n=10)$ and were compared to non-diabetic controls. Left ventricular function was measured by pressure-volume loops after 8 weeks of diabetes mellitus. The parameters for systolic function were the end systolic pressure-volume relationship (ESPVR) and the left ventricular end systolic pressure. The parameters for diastolic function were the left ventricular end diastolic pressure and the end diastolic pressure-volume relationship (EDPVR). Cardiac tissue was analysed by ELISA for the protein content of the cytokines TNF- $\alpha$, IL6, IL1- $\beta$, and TGF- $\beta 1$. Phosphorylation of MAPK p38 was analysed by western blot, and the total cardiac collagen content was analysed by Sirius red staining.
\end{abstract}

D. Westermann $\cdot$ S. Rutschow $\cdot$ S. Van Linthout $\cdot$ A. Linderer

C. Bücker-Gärtner $\cdot$ M. Sobirey $\cdot$ A. Riad $\cdot$ M. Pauschinger

H.-P. Schultheiss $\cdot$ C. Tschöpe

Department of Cardiology and Pneumology,

Charité University Hospital,

Benjamin Franklin Campus,

Berlin, Germany

C. Tschöpe $(\bowtie)$

Department of Cardiology, Charité-Universitätsmedizin Berlin, Campus Benjamin Franklin, Hindenburgdamm 30,

12200 Berlin, Germany

e-mail: ctschoepe@yahoo.com
Results Left ventricular dysfunction was documented by impaired ESPVR and EDPVR. Cardiac cytokine levels and cardiac fibrosis were increased in diabetic animals compared to controls. Treatment with the p38 inhibitor normalised cardiac cytokine levels and improved systolic function, but did not change cardiac fibrosis and diastolic dysfunction compared to placebo.

Conclusions/interpretation Pharmacological inhibition of p38 MAPK prevents cardiac inflammation and attenuates left ventricular dysfunction in diabetic cardiomyopathy.

Keywords Cardiac inflammation .

Diabetic cardiomyopathy · 38 MAPK .

Pressure-volume loops · Type 1 diabetes mellitus
Abbreviations
EDPVR end diastolic pressure-volume relationship
ESP end systolic pressure
ESPVR end systolic pressure-volume relationship
LV left ventricular
MAPK mitogen-activated protein kinase
PV pressure-volume
STZ streptozotocin; placebo-treated STZ mice
STZP38 SB203580-treated STZ mice
LVEDP left ventricular end diastolic pressure

\section{Introduction}

Cardiovascular complications, including diabetic cardiomyopathy, are the major cause of fatalities in diabetes. Diabetic cardiomyopathy is a distinct entity independent of coronary artery disease $[1,2]$ despite earlier beliefs common in the diabetic population [3]. Experimental models of diabetes 
mellitus, such as streptozotocin (STZ)-induced type 1 diabetes mellitus, imitate the structural and cellular abnormalities of diabetic cardiomyopathy [4]. These abnormalities include, among others, cardiac fibrosis [5] and cardiac inflammation, which lead to left ventricular (LV) dysfunction, mediated mainly by the diabetic milieu (e.g. high glucose levels, oxidative stress, angiotensin II) $[2,4,6]$. Cardiac inflammation, not only in diabetic cardiomyopathy, but also in most kinds of heart failure, is accompanied by elevated cardiac protein levels of cytokines, such as TNF$\alpha$, IL1- $\beta$, IL6, and TGF- $\beta 1$. These elevated cytokine levels are not only present in the cardiac tissue of animal models [7], but also in the serum of diabetic patients [8]. Given that increased levels of cytokines correlate with cardiac failure in humans [9], cardiac inflammation might be important in diabetic patients, too.

Mitogen-activated protein kinases (MAPK), e.g. the p38 MAPK, play a pivotal role in the development of heart failure, including diabetic cardiomyopathy [10]. Via its signalling cascade, the p38 MAPK modulates genes that regulate myocyte apoptosis, cellular hypertrophy, cardiac fibrosis, and cardiac cytokine-mediated inflammation [11-13]. Because the p38 MAPK is not only upregulated and phosphorylated by ischaemia [14], but also, for example, by angiotensin II [15], oxidative stress [16], and high glucose levels [10], inhibition of p38 MAPK could be a potent new therapeutic option for treatment of diabetic cardiomyopathy.

We therefore conducted a study to investigate possible beneficial effects of a p38 MAPK inhibitor on cardiac fibrosis and cardiac inflammation, and therefore on LV function, using a well-established animal model of STZinduced diabetic cardiomyopathy.

\section{Materials and methods}

Animals and treatment

Diabetes mellitus was induced in 20 C57/BL6J mice (male, 20-25 g bodyweight; Charles River, Sulzfeld, Germany) by injection of STZ $(50 \mathrm{mg} / \mathrm{kg}$ i.p. for 5 days $)$ dissolved in $0.1 \mathrm{~mol} / 1$ sodium citrate, $\mathrm{pH} 4.5$, as described previously [4]. Vehicle-treated C57/BL6J mice (male mice, 3 months old; Charles River, Germany) served as non-diabetic controls $(n=10)$. In the diabetic groups, hyperglycaemia was confirmed $48 \mathrm{~h}$ after last STZ injection and at the end of the study, using a reflectance meter (Accutrend; Boehringer, Mannheim, Germany). Diabetic mice were treated with SB 203580 (1 mg/kg bodyweight daily; Sigma-Aldrich, Steinheim, Germany), an inhibitor of the p38 MAPK $(n=10)$, or with placebo $(n=10)$ for 8 weeks. SB203580-treated STZ mice and placebo-treated STZ mice were denoted STZP38 and STZ respectively. In a pilot study, we investigated LV function of control mice treated with the p38 inhibitor and did not find any significant changes; this was consistent with the ex vivo model of Gorog et al. [17]. The investigation conformed to the Guide for the Care and Use of Laboratory Animals published by the US National Institutes of Health (NIH Publication No. 85-23, revised 1985).

\section{Surgical procedures and haemodynamic measurements}

The animals were anaesthetised (thiopental; $125 \mathrm{mg} / \mathrm{kg}$ bodyweight, i.p.), intubated, and artificially ventilated. As recently described [18], a 1.4 F microconductance pressure catheter (ARIA SPR-719; Millar-Instruments, Houston, TX, USA) was positioned in the LV for continuous registration of LV pressure-volume (PV) loops in a closed-chest model. Calibration of the volume signal was obtained by hypertonic saline $(10 \%)$ wash-in technique [19]. Indices of cardiac function were derived from PV data obtained both at steady state and during transient preload reduction by occlusion of the abdominal vena cava. Systolic function was quantified by LV end-systolic pressure (ESP) $(\mathrm{mmHg})$, while $\mathrm{d} P / \mathrm{d} t \max (\mathrm{mmHg} / \mathrm{s})$ and the slope of the ESP-volume relationship (ESPVR), the end arterial elastance, were used as an index of LV contractility. Diastolic function was measured by LV end-diastolic pressure (LVEDP) $(\mathrm{mmHg}), \mathrm{d} P / \mathrm{d} t \min (\mathrm{mmHg} / \mathrm{s})$, the isovolumetric relaxation Tau (ms), and end diastolic pressure-volume relationship (EDPVR), which is an indicator of LV stiffness, as determined from an exponential fit to the end-diastolic pressure-volume points. Global cardiac function was quantified by the end systolic and end diastolic volumes $(\mu \mathrm{l})$, stroke volume $(\mu \mathrm{l})$, ejection fraction $(\%)$ and heart rate (beats/min). After the experiment, the hearts were excised and weighed. Transverse sections (1-mm thick) of the hearts were rapidly frozen in liquid nitrogen, embedded in tissue tec (Sakura, Zoeterwoude, the Netherlands), and stored at $-80^{\circ} \mathrm{C}$ for Sirius red staining. The remaining LV was divided in two parts for ELISA and western blot measurements, rapidly frozen in liquid nitrogen, and stored at $-80^{\circ} \mathrm{C}$.

Western blot for evaluation of total p38 MAPK and p38 phosphorylation

LV samples were homogenised in lysis buffer containing proteinase inhibitor. An equal amount of protein was loaded into a $10 \%$ SDS-polyacrylamide gel. Total p38 MAPK (Cell Signaling Technology, Danvers, MA, USA), phosphorylated p38 MAPK (Cell Signaling Technology), and $\beta$ tubulin (Santa Cruz Biotechnology, Santa Cruz, CA, USA) were detected with each specific antibody. The 
blots were visualised with a chemiluminescene system (Amersham Bioscience, Buckinghamshire, UK). Quantitative analysis of the intensity of the bands was performed with NIH Image 1.63 software (National Institutes of Health, Bethesda, MD, USA).

\section{Cardiac TNF- $\alpha$, IL6, IL1- $\beta$, and TGF- $\beta$ ELISA}

TNF- $\alpha$, IL6, IL1- $\beta$, and TGF- $\beta$ were determined by ELISA (Pharmingen/BD Biosciences, San Diego, CA, USA) for myocardial tissue according to the manufacturer's instructions. All assays were performed twice, and the absorbance values were equalised with the absorbance values of glyceraldehyde-3-phosphate dehydrogenase (Active Motif, Carlsbad, CA, USA).

Immunohistochemistry

The total collagen content of the Sirius red (Polyscience, Warrington, PA, USA) stained sections was measured under circularly polarised light as recently reported [4]. It was then quantified by digital image analysis as the percentage of area fraction.

Statistical analyses

All data are expressed as mean \pm SEM. Statistical significance between multiple groups was determined using ANOVA and post hoc analysis with Student's $t$-test. Correlation between TNF- $\alpha$ and $\mathrm{d} P / \mathrm{d} t$ max was analysed using Pearson's correlation. Values of $p<0.05$ were considered significant.

\section{Results}

Characterisation of animal groups

Body weight and heart weight decreased in both STZ groups to a similar degree compared to the non-diabetic controls (body weight: STZP38 $18 \pm 3$ and STZ $17 \pm 4 \mathrm{~g}$ vs controls $31 \pm 3 \mathrm{~g},{ }^{*} p<0.05$; heart weight: STZP38 $95 \pm 3$ and STZ $90 \pm 2 \mathrm{mg}$ vs controls $138 \pm 3 \mathrm{mg}, * p<0.05)$. The calculated heart:body weight ratio showed no significant differences between the groups (STZP38 $5.2 \pm 0.2 \mathrm{mg} / \mathrm{g}$, STZ $5.0 \pm 0.2 \mathrm{mg} / \mathrm{g}$, controls $4.6 \pm 0.3 \mathrm{mg} / \mathrm{g}$, NS). Blood glucose levels were markedly increased in the STZP38 and STZ groups $48 \mathrm{~h}$ after the last STZ injection $(21.9 \pm 0.6 \mathrm{vs}$ $22.1 \pm 1 \mathrm{mmol} / \mathrm{l}$; NS) and 8 weeks after STZ injection $(29.8+1.7$ vs $31.5+2.3 \mathrm{mmol} / \mathrm{l}$; NS). Only animals with blood glucose levels over $20 \mathrm{mmol} / 148 \mathrm{~h}$ after STZ injection and $27 \mathrm{mmol} / 18$ weeks after STZ injection were used in the current study. Three animals in each STZ group did not reach these blood glucose levels and were excluded from this study. No side effects due to treatment with the p38 inhibitor were documented.

Haemodynamic data

Systolic function was impaired after 8 weeks of STZinduced diabetes mellitus in the STZ group compared to controls, with a significant decrease in ESP $(-43 \%), \mathrm{d} P / \mathrm{d} t$ $\max (-47 \%)$, and ESPVR $(-44 \%)$. Treatment with the MAPK inhibitor p38 led to a significant improvement of systolic function when STZP38 was compared to STZ, with an increase in ESP $(+24 \%), \mathrm{d} P / \mathrm{d} t$ max $(+26 \%)$, and ESPVR $(+34 \%)$. Nevertheless, treatment with p38 did not normalise systolic function in STZP38 compared to controls, resulting in a decrease in $\operatorname{ESP}(-33 \%), \mathrm{d} P / \mathrm{d} t \max (-35 \%)$, and ESPVR (-16\%) (Table 1).

Diastolic function was worse 8 weeks after STZ-induced diabetic cardiomyopathy, with an increase in LVEDP $(+74 \%)$, and EDPVR $(+65 \%)$, as compared to controls. This was accompanied by a decrease in $\mathrm{d} P / \mathrm{d} t \min (-39 \%)$ and a prolongation in Tau $(+21 \%)$. Treatment with the MAPK inhibitor p38 did not significantly affect this impairment of diastolic function (Table 1).

The heart rate was significantly decreased in both STZ groups as compared to controls. None of the parameters for ejection fraction, stroke volume, end systolic volume, or end diastolic volume were significantly different between any of the groups (Table 1).

Phosphorylation of MAPK p38

The activation state of the MAPK p38 was evaluated in the cardiac tissue of controls, STZ, and STZP38 animals by means of the ratio of phosphorylated p38 to total p38. In the cardiac tissue of diabetic mice, the phosphorylated p38: total p38 ratio was increased 1.5 -fold against controls $(p<0.05)$, whereas pharmacological inhibition with the p38 inhibitor in STZ resulted in a normalised activation state compared to controls (phosphorylated p38: control: 1,108 \pm 182 , STZ: 1,500 \pm 30 , STZP38: $811 \pm 127$ arbitrary units; total p38: control: 1,921 \pm 260 , STZ: 1,736 \pm 118 , STZP38: 1,405 \pm 81 arbitrary units). Total p38 towards $\beta$ tubulin was not significantly different between the groups (total p38/ $\beta$ tubulin ratio: control: $0.79 \pm 0.12$, STZ: $0.82 \pm 0.078$, STZP38: 0.81 \pm 0.089 ) (Fig. 1).

Cardiac TNF- $\alpha$, IL6, IL1- $\beta$, and TGF- $\beta$ levels

Relative to controls, the cardiac protein levels of proinflammatory cytokines in STZ diabetic mice were significantly higher: IL1- $\beta$ up $175 \%$, IL6 up $110 \%$, TNF- $\alpha$ up $46 \%$, and TGF- $\beta$ up $85 \%$, (all $p<0.05$ ). Treatment with $\mathrm{p} 38$ 
Table 1 Left ventricular haemodynamic function and cardiac fibrosis

\begin{tabular}{|c|c|c|c|}
\hline & Control & $\mathrm{STZ}$ & STZP38 \\
\hline \multicolumn{4}{|l|}{ Systolic LV function } \\
\hline LVP $(\mathrm{mmHg})$ & $112.3 \pm 7.3$ & $65.8 \pm 3.5^{* *}$ & $85.2 \pm 3.2 * * *$ \\
\hline $\mathrm{d} P / \mathrm{d} t \max (\mathrm{mmHg} / \mathrm{s})$ & $6,488 \pm 503$ & $3,643 \pm 285^{* *}$ & $4,845 \pm 564 * *, *$ \\
\hline $\mathrm{EES}(\mathrm{mmHg} / \mu \mathrm{l})$ & $1.75 \pm 0.1$ & $0.98 \pm 0.2 * *$ & $1.5 \pm 0.1 * *, *$ \\
\hline \multicolumn{4}{|l|}{ Diastolic LV function } \\
\hline LVEDP (mmHg) & $3.1 \pm 0.3$ & $12.7 \pm 3.2 * *$ & $10.6 \pm 2.1^{* *}$ \\
\hline $\mathrm{d} P / \mathrm{d} t \min (\mathrm{mmHg} / \mathrm{s})$ & $-5420 \pm 429$ & $-3164 \pm 782 * *$ & $-3664 \pm 288^{* *}$ \\
\hline Diastolic stiffness & $0.04 \pm 0.01$ & $0.11 \pm 0.02 * *$ & $0.01 \pm 0.01 * *$ \\
\hline \multicolumn{4}{|l|}{ Global LV function } \\
\hline Heart rate (beats/min) & $473 \pm 31$ & $323 \pm 23 * *$ & $356 \pm 15^{* *}$ \\
\hline Ejection fraction $(\%)$ & $62.5 \pm 6.5$ & $41.3 \pm 8.9$ & $45 \pm 6$ \\
\hline Stroke volume $(\mu \mathrm{l})$ & $36.1 \pm 1.5$ & $26.3 \pm 3.1$ & $30.1 \pm 4.1$ \\
\hline Volume end diastolic $(\mu \mathrm{l})$ & $59.4 \pm 5$ & $73.2 \pm 8$ & $66.8 \pm 7$ \\
\hline Volume end systolic $(\mu \mathrm{l})$ & $22.8 \pm 6$ & $44 \pm 11$ & $35.4 \pm 9$ \\
\hline \multicolumn{4}{|l|}{ Cardiac fibrosis } \\
\hline Sirius red staining $(\% \mathrm{AF})$ & $0.3 \pm 0.03$ & $0.81 \pm 0.1 * *$ & $0.72 \pm 0.08 * *$ \\
\hline
\end{tabular}

Values are means \pm SEM; results from immunohistological stainings with Sirius, measuring total collagen levels in $\%$ of area fraction (AF), are also shown.

LVP Left ventricular pressure, EES end arterial elastance, LVEDP left ventricular end diastolic pressure.

${ }^{*} p<0.05$ for difference from STZ

$* * p<0.05$ for difference from controls

normalised IL1- $\beta$, TNF- $\alpha$, and TGF- $\beta$ in STZP38, but only partly reduced IL6 levels compared to controls. Thus IL6 was significantly reduced in STZ compared to STZP38 $(-24 \%, p<0.05)$, but remained increased when STZP38 $(+40 \%, p<0.05)$ was compared to controls (Fig. 2). Furthermore, the cytokine levels of TNF- $\alpha$ showed a significant negative correlation with the haemodynamic parameter $\mathrm{d} P /$ $\mathrm{d} t$ max, when data of both diabetic groups for TNF- $\alpha$ and $\mathrm{d} P / \mathrm{d} t$ max were correlated $\left(r^{2}=0.57, p<0.05\right)$.

Total collagen content

The total collagen measured as the percentage of area fraction was increased 2.7-fold in STZ and 2.4-fold in STZP38 compared to controls. P38 inhibition did not significantly alter the total collagen content when STZP38 was compared to STZ (Fig. 3, Table 1).

\section{Discussion}

The novel finding of our study was that pharmacological inhibition of the p38 MAPK improves LV function in STZ-induced diabetic cardiomyopathy, an improvement associated with reduced cardiac inflammation, characterised by reduced myocardial pro-inflammatory cytokine levels.
Cardiac inflammation

Diabetic cardiomyopathy is associated with increased levels of the pro-inflammatory cytokines TNF- $\alpha$ and IL1- $\beta$ [7]. This cardiac inflammation is linked with decreased LV function, not only in diabetic cardiomyopathy, but also for example in myocardial infarction, pressure overload, and dilated cardiomyopathy [20-22]. Increased cytokines are one of the mechanisms leading to LV dysfunction in diabetes mellitus, as demonstrated by an inverse correlation between $\mathrm{d} P / \mathrm{d} t \max$ and TNF- $\alpha$ in diabetic animals in this study. Furthermore, not only the cardiac protein contents of TNF- $\alpha$ and IL1- $\beta$ were increased in our study, but also other pro-inflammatory cytokines, such as TGF- $\beta$ and IL6.

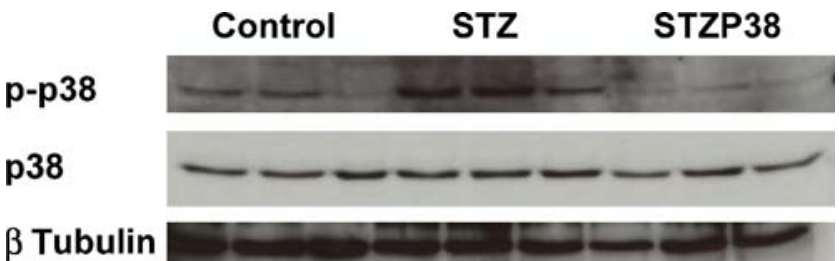

Fig. 1 Representative western blots for control, STZ, and STZP38 mice, showing phosphorylated p38 (p-p38), total p38 (p38), and $\beta$ tubulin. STZ-induced diabetes mellitus does not affect total p38 but significantly up-regulates p-p38, revealing an increased activation state of MAPK p38. Pharmacological inhibition of p38 downregulates p-p38 to non-diabetic control levels 
Fig. 2 Data representing cardiac cytokine levels, as measured by ELISA, showing significantly increased TNF- $\alpha$ (a), IL6 (b), IL1- $\beta$ (c), and TGF- $\beta$ (d) in STZ compared to controls. In STZP38 compared with controls, TNF- $\alpha$, IL1- $\beta$, and TGF- $\beta$ were normalised, whereas IL6 was only reduced by treatment with $\mathrm{p} 38$ inhibitor. Asterisk: $p<0.05$ for difference
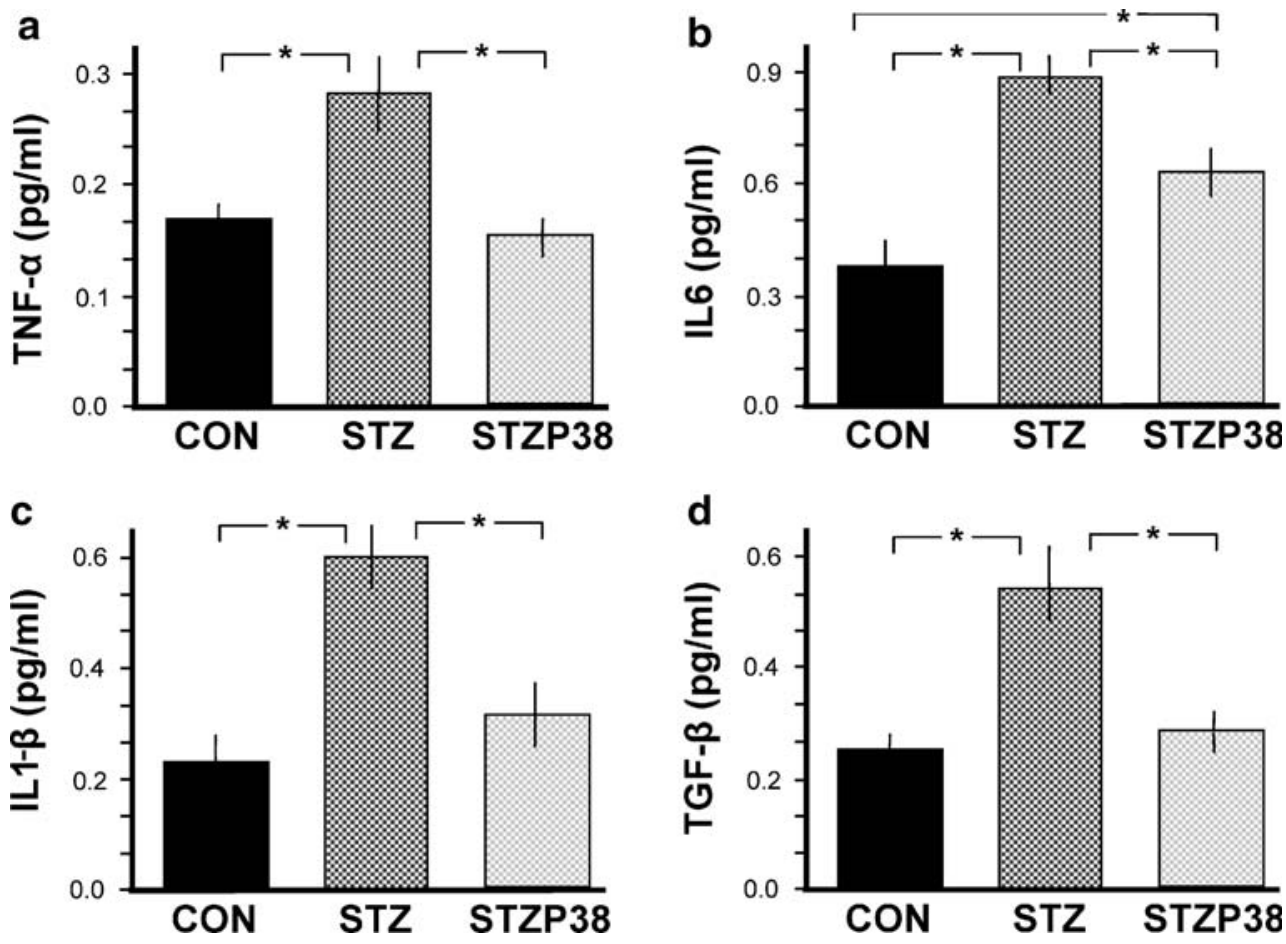

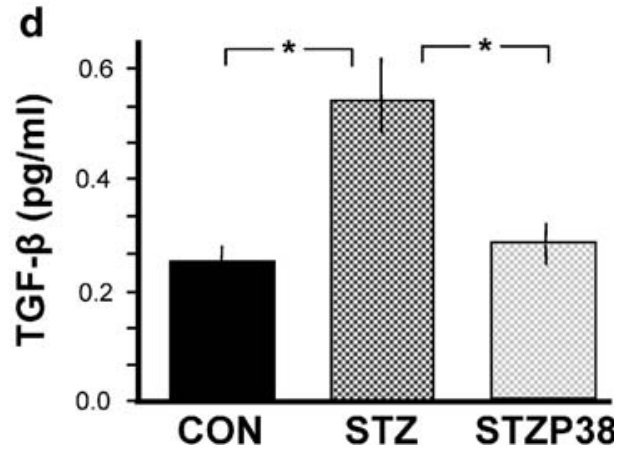

Cytokines attenuate myocyte contractility, achieving this directly through the reduction of systolic cytosolic calcium via alterations in sarcoplasmic reticulum function and indirectly by down-regulating sarcoplasmic calcium ATPase expression, as reviewed [22]. Inflammatory cytokines were originally thought to be produced only by circulating lymphocytes, but recent evidence suggests a direct role of cardiomyocytes in pro-inflammatory cytokine production $[23,24]$.

The p38 MAPK is a key regulatory pathway for many genes, including genes regulating TNF- $\alpha$, IL1- $\beta$, and TGF- $\beta$ [25] in a wide variety of cell types. The diabetic milieu upregulates p38 activity by phosphorylation [10]. Furthermore, p38 activity is intensified by TNF- $\alpha$ in cultured cardiomyocytes, as a positive feedback loop [13]. The p38 inhibitor prevents this phosphorylation of the p38 MAPK in cardiac tissue, as shown in the current study (Fig. 1), thereby inhibiting its function [26]. The signalling pathways dependent on p38 MAPK should be analysed in further studies.

Inhibited phosphorylation of the p38 MAPK normalised the cytokine levels of TNF- $\alpha$, IL1- $\beta$, and TGF- $\beta$ and reduced the levels of IL6 in the cardiac tissue of our diabetic animals compared to controls, which is in agreement with Peng et al. [27] (Fig. 2). A recently conducted study [13] on non-diabetic, transgenic, p38overexpressing mice that develop impaired cardiac function and increased cytokine levels found that these mice show an improvement of LV function after p38 inhibition. The mice also showed a reduction in circulating cytokine levels, despite a further increase in cardiac cytokine levels. These
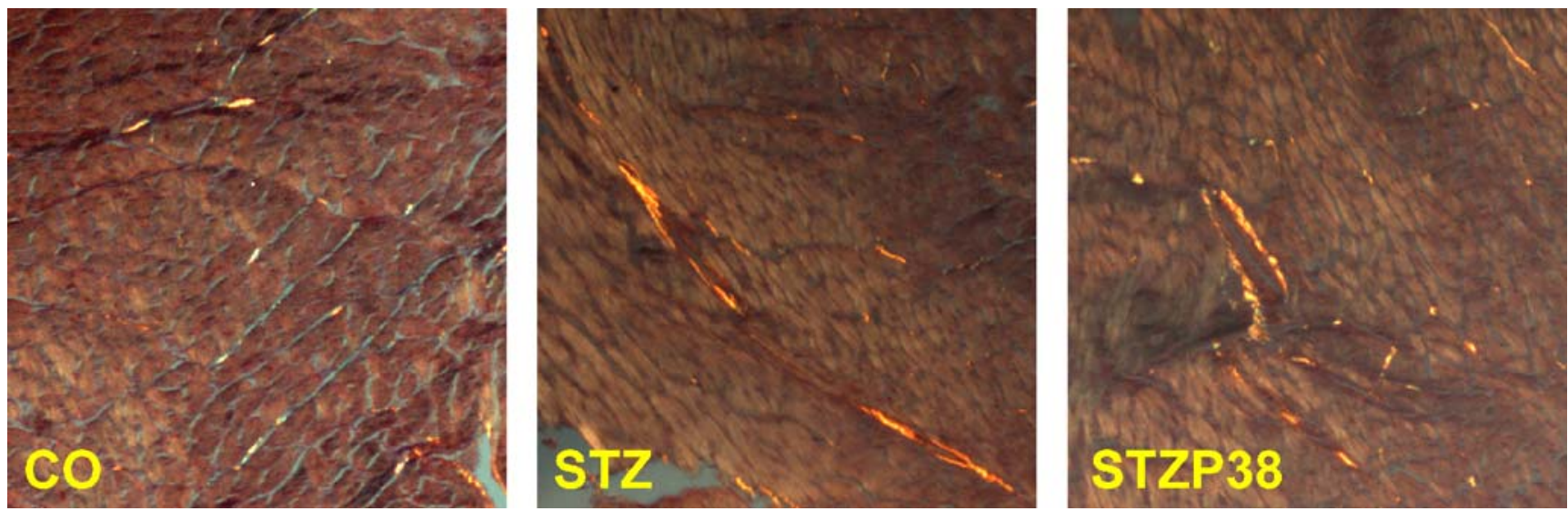

Fig. 3 Sirius Red staining (original magnification $\times 100)$ in representative heart sections of controls $(\mathrm{CO})$, STZ, and STZP38, showing significantly increased myocardial fibrosis in STZ and STZP38 compared to controls 
discrepancies might be explained by the transgenic animal model, the non-diabetic status of the animals, and/or by the different p38 inhibitor used. Further studies should evaluate the circulating plasma levels in STZ-induced diabetes mellitus.

In our study, the LV systolic function of STZP38 was enhanced compared to STZ. End arterial elastance (a loadindependent marker for systolic contractility), $\mathrm{d} P / \mathrm{d} t \max$, and the ESP were all improved. Reduced cardiac inflammation improves LV systolic function. Nevertheless, multiple variables affecting LV function exist, which explains why normalised inflammation alone does not automatically lead to normalised systolic function. One of the other variables affecting cardiac function is cardiac fibrosis.

\section{Cardiac fibrosis}

Tissue fibrosis is a pathological hallmark of diabetic complications [28]. Diabetic cardiomyopathy is characterised by increased extracellular matrix deposition of collagen in the interstitium of diabetic hearts [28, 29]. Accordingly, the total collagen content in the cardiac tissue of diabetic animals was increased compared to controls. Enhanced cardiac fibrosis has a direct impact not only on LV systolic function, but also especially on diastolic function [30]. This impact is revealed by the increased cardiac stiffness (the load-independent parameter for cardiac stiffness in diastole) and by the increased LVEDP (the load-dependent parameter for diastolic filling pressure) in our diabetic animals, thus indicating diastolic dysfunction in diabetic cardiomyopathy.

Interestingly, treatment with the p38 MAPK inhibitor did not reduce fibrosis in STZP38 compared to the STZ group (Fig. 3). Consistent with these findings, diastolic function was still impaired with increased cardiac stiffness and increased LVEDP in STZP38. These findings are unexpected, because it has been shown that $\mathrm{p} 38$ inhibition leads to reduced cardiac fibrosis in a transgenic mouse model with enhanced p38 activity [13] and reduces LV dilatation after myocardial infarction in rats [31].

The aetiology of diabetes-induced cardiac fibrosis is multifactoral. Factors leading to cardiac fibrosis include: TGF- $\beta$ [32], focal changes in microvessels leading to ischaemia [33], non-enzymatic glycosylation and lysyloxidase-mediated cross-linking [34], high glucose levels, and pro-fibrotic-acting peptides such as aldosterone and angiotensin II. It is therefore intriguing to speculate that inhibition of $\mathrm{p} 38$, with normalisation of the pro-inflammatory and pro-fibrotic cytokines TNF- $\alpha$, IL1- $\beta$ and TGF- $\beta$ alone, is not potent enough to reduce fibrosis in vivo in this animal model of diabetes mellitus, especially since the $\mathrm{p} 38$ inhibitor normalised the activation state of p38 to a nondiabetic level. IL6 was only partly reduced, but not normalised, which may lead then to the above-mentioned reasons for unchanged cardiac fibrosis.

Conclusions and clinical implications

Pharmacological inhibition of the p38 MAPK improves LV function in STZ-induced diabetic cardiomyopathy. This improvement is associated with reduced cardiac inflammation, but does not change cardiac fibrosis. In conclusion, these findings show that activation of $\mathrm{p} 38$ is involved in the development of diabetic cardiomyopathy. Further studies should investigate whether $\mathrm{p} 38$ inhibition could serve as a new therapeutic target for the treatment of diabetic cardiomyopathy in addition to conventional anti-diabetic therapy.

Acknowledgements This study was supported by the Deutsche Forschungsgesellschaft (SFB-TR-19; B5). The authors would like to thank M. Hanna for proof-reading the final version of the manuscript.

\section{References}

1. Spector KS (1998) Diabetic cardiomyopathy. Clin Cardiol 21:885-887

2. Poornima IG, Parikh P, Shannon RP (2006) Diabetic cardiomyopathy: the search for a unifying hypothesis. Circ Res 98:596-605

3. Bell DS (2003) Diabetic cardiomyopathy. Diabetes Care 26: 2949-2951

4. Tschope C, Walther T, Koniger J et al (2004) Prevention of cardiac fibrosis and left ventricular dysfunction in diabetic cardiomyopathy in rats by transgenic expression of the human tissue kallikrein gene. Faseb J 18:828-835

5. Asbun J, Villarreal FJ (2006) The pathogenesis of myocardial fibrosis in the setting of diabetic cardiomyopathy. J Am Coll Cardiol 47:693-700

6. Tschope C, Spillmann F, Rehfeld U et al (2004) Improvement of defective sarcoplasmic reticulum $\mathrm{Ca}^{2+}$ transport in diabetic heart of transgenic rats expressing the human kallikrein-1 gene. Faseb J 18:1967-1969

7. Tschope C, Walther T, Escher F et al (2005) Transgenic activation of the kallikrein-kinin system inhibits intramyocardial inflammation, endothelial dysfunction, and oxidative stress in experimental diabetic cardiomyopathy. Faseb J 14:2057-2059

8. Azar ST, Salti I, Zantout MS, Major S (2000) Alterations in plasma transforming growth factor beta in normoalbuminuric type 1 and type 2 diabetic patients. J Clin Endocrinol Metab 85:4680-4682

9. Torre-Amione $\mathrm{G}$ (2005) Immune activation in chronic heart failure. Am J Cardiol 95:3C-8C (discussion 38C-40C)

10. Adhikary L, Chow F, Nikolic-Paterson DJ et al (2004) Abnormal p38 mitogen-activated protein kinase signalling in human and experimental diabetic nephropathy. Diabetologia 47:1210-1222

11. Mackay K, Mochly-Rosen D (1999) An inhibitor of p38 mitogenactivated protein kinase protects neonatal cardiac myocytes from ischemia. J Biol Chem 274:6272-6279

12. Wang Y, Huang S, Sah VP et al (1998) Cardiac muscle cell hypertrophy and apoptosis induced by distinct members of the p38 mitogen-activated protein kinase family. J Biol Chem 273:2161-2168

13. Li M, Georgakopoulos D, Lu G et al (2005) p38 MAP kinase mediates inflammatory cytokine induction in cardiomyocytes and 
extracellular matrix remodeling in heart. Circulation 111: 2494-2502

14. Ma XL, Kumar S, Gao F et al (1999) Inhibition of p38 mitogenactivated protein kinase decreases cardiomyocyte apoptosis and improves cardiac function after myocardial ischemia and reperfusion. Circulation 99:1685-1691

15. Fischer TA, Ludwig S, Flory E et al (2001) Activation of cardiac c-Jun $\mathrm{NH}(2)$-terminal kinases and p38-mitogen-activated protein kinases with abrupt changes in hemodynamic load. Hypertension 37:1222-1228

16. Zhang GX, Kimura S, Nishiyama A, Shokoji T, Rahman M, Abe Y (2004) ROS during the acute phase of Ang II hypertension participates in cardiovascular MAPK activation but not vasoconstriction. Hypertension 43:117-124

17. Gorog DA, Tanno M, Cao X et al (2004) Inhibition of p38 MAPK activity fails to attenuate contractile dysfunction in a mouse model of low-flow ischemia. Cardiovasc Res 61:123-131

18. Westermann D, Knollmann BC, Steendijk P et al (2006) Diltiazem treatment prevents diastolic heart failure in mice with familial hypertrophic cardiomyopathy. Eur J Heart Fail 8:115-121

19. Steendijk P, Staal E, Jukema JW, Baan J (2001) Hypertonic saline method accurately determines parallel conductance for dual-field conductance catheter. Am J Physiol Heart Circ Physiol 281: H755-H763

20. Steenbergen C (2002) The role of p38 mitogen-activated protein kinase in myocardial ischemia/reperfusion injury; relationship to ischemic preconditioning. Basic Res Cardiol 97:276-285

21. Meldrum DR (1998) Tumor necrosis factor in the heart. Am J Physiol 274:R577-R595

22. Nian M, Lee P, Khaper N, Liu P (2004) Inflammatory cytokines and postmyocardial infarction remodeling. Circ Res 94:1543-1553

23. Bazzoni F, Beutler B (1996) The tumor necrosis factor ligand and receptor families. N Engl J Med 334:1717-1725
24. Mann DL (2003) Stress-activated cytokines and the heart: from adaptation to maladaptation. Annu Rev Physiol 65:81-101

25. Saklatvala J (2004) The p38 MAP kinase pathway as a therapeutic target in inflammatory disease. Curr Opin Pharmacol 4:372-377

26. Lee JC, Kassis S, Kumar S, Badger A, Adams JL (1999) p38 mitogen-activated protein kinase inhibitors-mechanisms and therapeutic potentials. Pharmacol Ther 82:389-397

27. Peng T, Lu X, Lei M, Moe GW, Feng Q (2003) Inhibition of p38 MAPK decreases myocardial TNF-alpha expression and improves myocardial function and survival in endotoxemia. Cardiovasc Res 59:893-900

28. Shimizu M, Umeda K, Sugihara N et al (1993): Collagen remodelling in myocardia of patients with diabetes. J Clin Pathol 46:32-36

29. Fang ZY, Prins JB, Marwick TH (2004) Diabetic cardiomyopathy: evidence, mechanisms, and therapeutic implications. Endocr Rev 25:543-567

30. Zile MR, Brutsaert DL (2002) New concepts in diastolic dysfunction and diastolic heart failure: Part II: Causal mechanisms and treatment. Circulation 105:1503-1508

31. See F, Thomas W, Way K et al (2004) p38 mitogen-activated protein kinase inhibition improves cardiac function and attenuates left ventricular remodeling following myocardial infarction in the rat. J Am Coll Cardiol 44:1679-1689

32. Massague J, Chen YG (2000) Controlling TGF-beta signaling. Genes Dev 14:627-644

33. Yagihashi S, Wada R, Yamagishi S (2002) Diabetic microangiopathy: pathology and current understanding of its pathogenesis. Verh Dtsch Ges Pathol 86:91-100

34. Buckingham B, Reiser KM (1990) Relationship between the content of lysyl oxidase-dependent cross-links in skin collagen, nonenzymatic glycosylation, and long-term complications in type I diabetes mellitus. J Clin Invest 86:1046-1054 